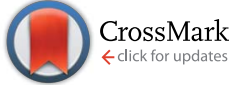

Cite this: J. Mater. Chem. A, 2016, 4, 18426

Received 8th September 2016 Accepted 4th November 2016

DOI: $10.1039 / c 6 t a 07772 b$

www.rsc.org/MaterialsA

\title{
Selective surface modification in bimodal mesoporous CMK-5 carbon $\uparrow$
}

\begin{abstract}
C. Weinberger, X. Cao and M. Tiemann*
Ordered, bimodal mesoporous CMK- 5 carbon is prepared by using mesoporous SBA-15 silica as a structural mold. The carbon material is chemically modified by oxidative treatment with acidic persulfate solution. This leads to the creation of oxygen-containing functionalities at the pore walls of the carbon (up to $13 \mathrm{wt} \%$ oxygen), as confirmed by IR spectroscopy. The oxidative treatment is carried out before removal of the silica mold which ensures that only one of the two distinct modes of mesopores (namely, the intratubular pores) is affected; the other mode (inter-tubular pores) is protected from oxidation by the presence of the silica mold. This is proven by water vapor physisorption analysis. The oxidatively treated (intra-tubular) pores are significantly more polar and, hence, better wettable than the untreated (intertubular) pores.
\end{abstract}

\section{Introduction}

Ordered mesoporous carbon materials with regular pore architectures and uniform pore sizes are frequently prepared by the structure replication (nanocasting) method, ${ }^{1-4}$ using mesoporous silica as structural molds. ${ }^{5,6}$ The carbon material is created inside the pores of the silica (e.g. by carbonization of sucrose or furfuryl alcohol). If the entire pore volume of the silica matrix is filled with carbon, then subsequent removal of the silica by chemical etching (using $\mathrm{NaOH}$ or HF solution) will yield an exact carbon 'replica' of the original silica pore system. For example, SBA-15 silica ${ }^{7}$ with its $2 \mathrm{D}$ hexagonal array of linear, cylindrical mesopores will yield mesoporous CMK-3 carbon, ${ }^{8}$ which basically consists of carbon rods in a $2 \mathrm{D}$ hexagonal parallel arrangement. By varying the synthesis parameters it is possible to not completely fill the pores of the silica matrix with carbon, but to create only a layer of carbon at the pore walls; the center of the cylindrical pores remains hollow. After removal of the silica matrix the resulting carbon material, frequently termed CMK- $5,{ }^{9,10}$ then consists of cylindrical rods that are hollow ('tubes'), therefore constituting two distinct types of mesopores: (i) inter-tubular pores (between adjacent rods; these pores are present in both CMK-3 and CMK-5) and (ii) intratubular pores (i.e., the hollow interior of the rods; this type of pores is missing in CMK-3).

In general, nanoporous carbon phases are promising materials for numerable applications, including in batteries, ${ }^{11-13}$ as capacitors, ${ }^{14,15}$ as sorbents for biomolecules, ${ }^{16,17}$ in

Department of Chemistry, University of Paderborn, Warburger Str. 100, D-33098 Paderborn, Germany.E-mail: michael.tiemann@upb.de

$\dagger$ Electronic supplementary information (ESI) available. See DOI: $10.1039 / \mathrm{c} 6 \mathrm{ta} 07772 \mathrm{~b}$ chromatography, ${ }^{18,19}$ wastewater cleaning, ${ }^{20,21}$ and catalysis. ${ }^{22-24}$ For the latter, porous carbon may serve as a support material for catalytically active species or carry organic functionalities covalently attached to the carbon framework. CMK-5 carbon, with its above-described bimodal mesoporosity, offers particular chances, since the two distinct pore modes can be addressed separately and independently, which opens up an opportunity to create materials with dual functionality. During the synthesis of CMK-5 the intra-tubular pores are formed upon carbonization of the precursor compound (furfuryl alcohol) within the silica mold. At this stage, the inter-tubular pores are still blocked by the silica matrix; they become accessible only after the removal of silica by chemical etching. This means that the intra-tubular pores can be modified selectively (before removal of silica) without affecting the inter-tubular pores (Scheme 1)..$^{25,26}$

This concept offers new opportunities, for example in the field of heterogeneous catalysis using bifunctional catalysts. Bifunctional catalysts combine two distinct types of catalytically active sites (guest species and/or functional groups) in one

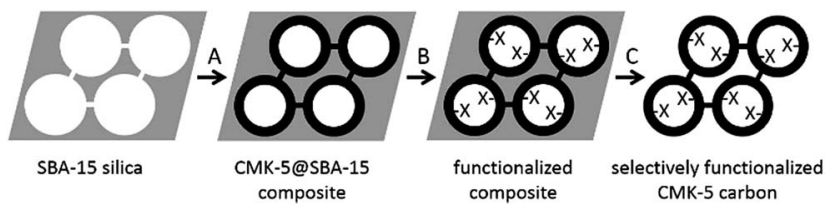

Scheme 1 Synthesis strategy for selective surface modification of bimodal mesoporous CMK-5 carbon. The carbon is prepared by coating the mesopores of SBA-15 silica (step A). The intra-tubular pores are selectively modified (with functionalities depicted as ' $X$ ', step B). By removal of the silica matrix (step C) a second pore mode (intertubular pores) is generated which is not surface-modified. 
material, thereby facilitating fast and effective catalytic one-pot reactions. Recent reports include mesoporous silica and/or carbon materials containing combinations of various kinds of catalyst species in their mesopore channels. ${ }^{27-34}$ Multiple functionalization is also interesting for phase-boundary catalysis, i.e. catalytic processes that involve reactants immiscible in the same solvent; ${ }^{35}$ single-phase catalysts which exhibit polar and nonpolar surfaces at the same time have been shown to improve such processes. ${ }^{36,37}$ For some catalytic reactions, however, it might be advantageous to immobilize the two active species in distinct pore systems of the same material, for example in order to prevent them from inactivating each other. ${ }^{38,39}$ This latter option is available with bimodal mesoporous CMK-5 carbon, as described above.

Here we present a straightforward method to functionalize the intra-tubular pores of CMK-5 carbon selectively without affecting the inter-tubular pore system. Acidic ammonium persulfate solution is used as an oxidation agent, selectively for the intra-tubular pores, while the inter-tubular pore system remains unchanged.

\section{Results and discussion}

Elemental carbon was created in the cylindrical pores of mesoporous SBA-15 silica by carbonizing furfuryl alcohol and oxalic acid as described in the Experimental section. The employed synthesis protocol leads to a coating of the pore walls with a homogeneous layer of carbon while the center of the pores remains hollow. ${ }^{9}$ After selective removal of the silica by etching with HF solution a mesoporous carbon material results which is frequently referred to as CMK-5. ${ }^{10}$ As mentioned in the Introduction, mesoporous CMK-5 is a particularly interesting material as it exhibits two distinct types (modes) of mesopores. It can be described as a regular array of hollow, tubular 'nanorods': (i) the voids between adjacent rods constitute one mode of mesopores; they originate from the removal of the silica matrix (and they also exist in other types of mesoporous carbon materials prepared by nanocasting, such as CMK-3). In the following, we will refer to this type of mesopores as 'inter-tubular pores'. (ii) In addition, since the rods in CMK-5 are hollow, a second mode of mesoporosity results, in the following denoted as 'intra-tubular pores'. These intra-tubular pores are unique in CMK-5; they already exist before removal of the SBA-15 silica matrix, i.e. in the CMK-5@SBA-15 composite material right after carbonization. Hence, the intra-tubular carbon pores can be modified (e.g. filled with guest species or functionalized at the pore walls) independently before the removal of the silica matrix (see Scheme 1), as will be shown in the following.

We have subjected the CMK-5@SBA-15 carbon/silica composite to oxidative treatment by dispersing the material in a persulfate solution and stirring at room temperature for a defined duration between $1 \mathrm{~h}$ and $10 \mathrm{~h}$. The oxidative treatment does not affect the long-range structural order of the final CMK-5 carbon materials, as confirmed by powder X-ray diffraction (see ESI section, Fig. S1 $\dagger$ ). This is worth noticing, since treatment under harsher oxidative conditions has previously been observed to compromise the structural integrity of mesoporous CMK-3 or CMK-5 carbon. ${ }^{\mathbf{4 0 - 4 2}}$ FT-IR spectra of the (silica-free) CMK-5 carbon samples treated for $0 \mathrm{~h}, 1 \mathrm{~h}, 6 \mathrm{~h}$, and $10 \mathrm{~h}$, respectively, are shown in Fig. 1. For a semi-quantitative analysis the spectra were normalized with respect to the intensity of the band at $c a .1582 \mathrm{~cm}^{-1}$ (aromatic C-C ring stretching mode $\mathrm{es}^{\mathbf{4}-45}$ ). The oxidative treatment results in the occurrence of a band at ca. $1730 \mathrm{~cm}^{-1}$ which increases in intensity upon longer treatment. This band can be attributed to the carbonyl $\mathrm{C}=\mathrm{O}$ stretching mode in a keto or carboxyl function. ${ }^{43-45}$ Likewise, a band at ca. $1220 \mathrm{~cm}^{-1}$ also appears upon oxidation; this corresponds presumably to a $\mathrm{C}-\mathrm{O}$ stretching mode. ${ }^{\mathbf{4 4 , 4 5}}$ (The band at ca. $1150 \mathrm{~cm}^{-1}$ is assigned to a $\mathrm{C}-\mathrm{C}$ stretching mode. ${ }^{43-45}$ ) Hence, it can be concluded that the treatment with persulfate solution creates oxygen-containing polar surface functions (which, in turn, leads to an increase in hydrophilicity, as will be shown below). This is confirmed by elemental analysis. The initial oxygen content in the untreated CMK-5 carbon material is $3.8 \mathrm{wt} \%$; the value gradually increases to $13.0 \mathrm{wt} \%$ after 10 hours of oxidative treatment (see Table 1). It should be noted again that only the intra-tubular pores were modified, since the oxidative treatment was performed before the removal of the silica phase. The inter-tubular pores of CMK-5 carbon were not accessible to the persulfate solution at this stage.

Fig. 2 (left) shows the $\mathrm{N}_{2}$ physisorption isotherms of the (silica-free) CMK-5 samples that previously (i.e. before removal of the silica) underwent oxidative treatment for $0 \mathrm{~h}, 1 \mathrm{~h}, 6 \mathrm{~h}$, and $10 \mathrm{~h}$, respectively. All isotherms exhibit a shape of the $\operatorname{IV}(\mathrm{a})$ type, ${ }^{46}$ indicating uniform mesopores. The $\mathrm{BJH}$ pore size distribution curves (Fig. 2, right) reveal an asymmetric peak between $c a .3 .0 \mathrm{~nm}$ and $4.5 \mathrm{~nm}$. This peak is attributable to both modes of mesopores, the inter-tubular pores with an average diameter of $c a .4 .0 \mathrm{~nm}$ and the intra-tubular pores with an average diameter of $c a .3 .5 \mathrm{~nm}$. The two pore modes are not resolved in the pore size distribution curves, but the existence of the intra-tubular pores $(3.5 \mathrm{~nm})$ will be substantiated below (Fig. 4); they are responsible for the tailing of the asymmetric peak on the left-hand side. Oxidative treatment leads to a slight

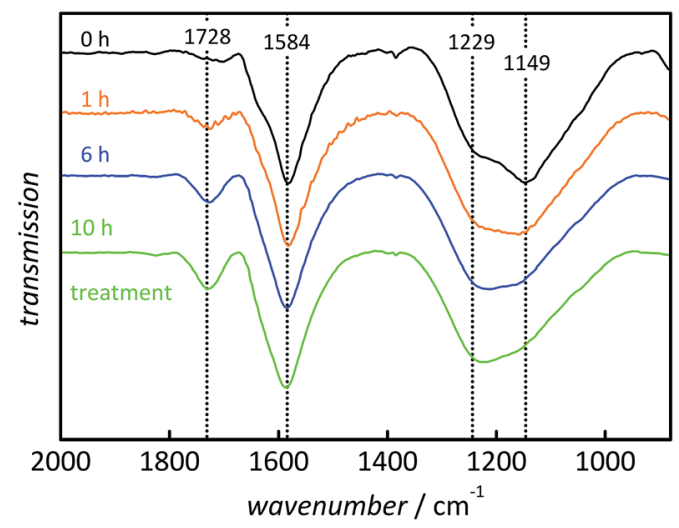

Fig. 1 FT-IR spectra of CMK-5 carbon before and after oxidative treatment with persulfate solution up to $10 \mathrm{~h}$. Spectra are normalized to the intensity of the band at $1582 \mathrm{~cm}^{-1}$ (aromatic $\mathrm{C}-\mathrm{C}$ ring stretching mode ${ }^{43-45}$ ). 
Table 1 Structural properties of CMK-5 carbon and CMK-5 CSBA-15 composites before and after oxidative treatment with persulfate solution up to $10 \mathrm{~h}$

\begin{tabular}{|c|c|c|c|c|c|c|}
\hline Sample & Treatment & $A_{\mathrm{BET}}{ }^{a} \mathrm{~m}^{2} \mathrm{~g}^{-1}$ & $V^{b} \mathrm{~cm}^{3} \mathrm{~g}^{-1}$ & \multicolumn{2}{|c|}{$D_{\mathrm{BJH}}{ }^{c} \mathrm{~nm}$} & Weight $\%$ oxygen \\
\hline \multirow[t]{3}{*}{ CMK-5 carbon } & (None) & 1084 & 1.55 & - & 4.0 & 3.8 \\
\hline & $1 \mathrm{~h}$ & 1057 & 1.49 & - & 4.0 & 7.1 \\
\hline & $10 \mathrm{~h}$ & 1031 & 1.43 & - & 4.0 & 13.0 \\
\hline SBA-15 silica & (None) & 457 & 1.36 & $(-)$ & 8.6 & $(-)^{d}$ \\
\hline \multirow{2}{*}{ CMK-5@SBA-15 composite } & (None) & 273 & 0.33 & 3.6 & 7.2 & $(-)^{d}$ \\
\hline & $10 \mathrm{~h}$ & 160 & 0.24 & 3.5 & 7.3 & $(-)^{d}$ \\
\hline
\end{tabular}

${ }^{a}$ Specific surface area. ${ }^{b}$ Specific pore volume. ${ }^{c}$ Pore diameter(s). ${ }^{d}$ (Not measured).

decrease of the specific pore volume to $c a$. $90 \%$ of its initial value (from $1.55 \mathrm{~cm}^{3} \mathrm{~g}^{-1}$ to $1.39 \mathrm{~cm}^{3} \mathrm{~g}^{-1}$ ); the BET surface area reduces to $c a$. $89 \%$ (from $1073 \mathrm{~m}^{2} \mathrm{~g}^{-1}$ to $954 \mathrm{~cm}^{2} \mathrm{~g}^{-1}$ ). The decrease in pore volume and BET surface area (both normalized to the sample mass) can be explained by the increase in sample mass as a consequence of the creation of oxygen-containing surface functions. The average pore sizes, however, do not change significantly and the pore size distribution does not become any broader. These findings confirm that the oxidative treatment does not affect the structural characteristics of the CMK-5 material. It should be noted that the samples exhibit micropores only to a very small extent, as commonly observed for mesoporous carbon prepared from furfuryl alcohol.,9,10 (Micropores contribute to the BET surface area by only $11 \mathrm{~m}^{2}$ $\mathrm{g}^{-1}$ in the untreated sample and by $50 \mathrm{~m}^{2} \mathrm{~g}^{-1}$ in the sample treated for $10 \mathrm{~h}$.) Microporosity will therefore be neglected in the following.

Water vapor sorption analysis reveals more significant differences between oxidatively treated and untreated CMK-5 samples than $\mathrm{N}_{2}$ sorption analysis does. Fig. 3 shows the respective water sorption isotherms $\left(20^{\circ} \mathrm{C}\right)$ for the same
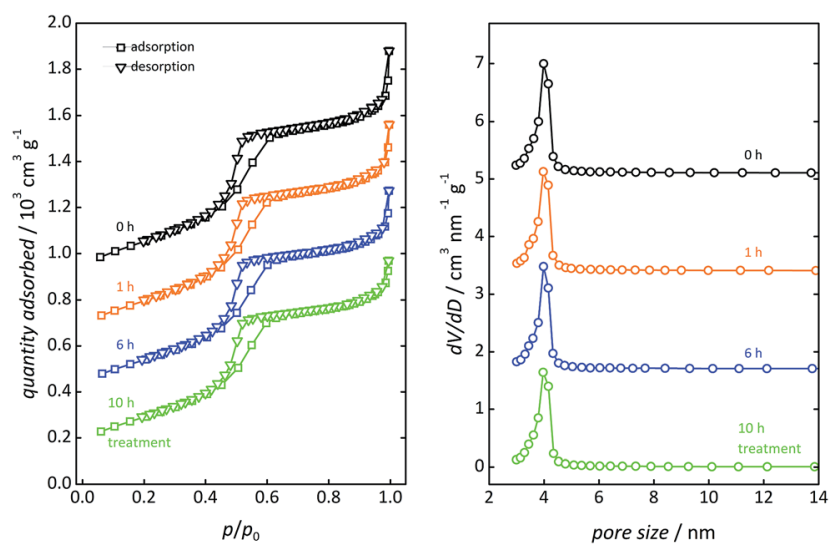

Fig. $2 \mathrm{~N}_{2}$ physisorption isotherms (left, vertically shifted in steps of $0.25 \times 10^{3} \mathrm{~cm}^{3} \mathrm{~g}^{-1}$ each) and $\mathrm{BJH}$ pore size distribution curves (right) of CMK-5 carbon before and after oxidative treatment with persulfate solution up to $10 \mathrm{~h}$. samples as in Fig. 2. All isotherms show a clear type- $\mathrm{V}$ behavior, ${ }^{46}$ as commonly observed for this kind of mesoporous carbon. ${ }^{47}$ This isotherm shape is characterized by weak interaction between the sorbent (carbon) and the sorptive $\left(\mathrm{H}_{2} \mathrm{O}\right)$. Almost no adsorption occurs at low relative pressure and a steep incline due to pore filling takes place at relative pressures significantly higher than those for $\mathrm{N}_{2}$ sorption. Strong hysteresis is observed upon desorption which is attributed to different mechanisms of filling and emptying of the mesopores. Recent studies suggest that the mechanism of pore filling is marked by the formation of adsorbed water clusters. ${ }^{47-50}$ For the CMK-5 carbon samples both the steep incline of the adsorption isotherm and the steep decline of the desorption isotherm occur at variable relative pressure, depending on the relative amount of polar surface functions resulting from oxidative treatment for variable duration. For non-functionalized CMK-5 carbon the mesopore filling occurs at $c a . p / p_{0}=0.83$ (adsorption

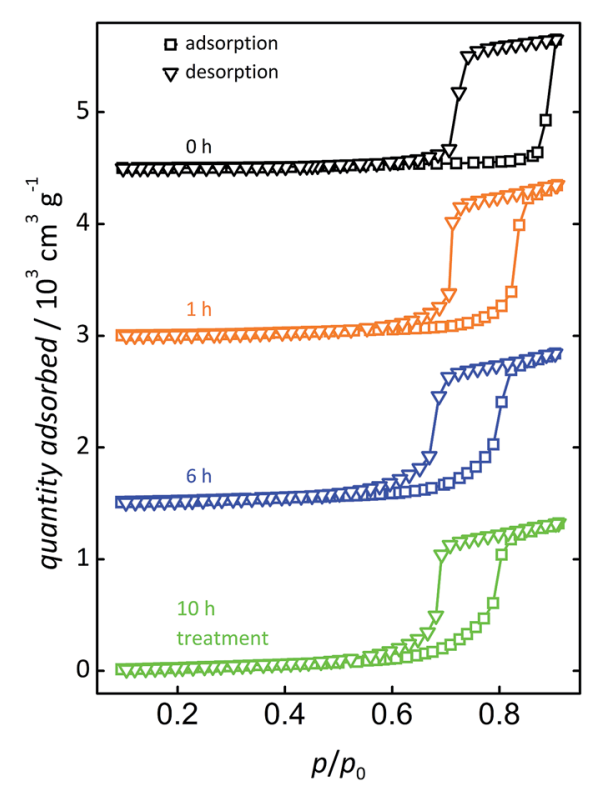

Fig. 3 Water vapor physisorption isotherms $\left(20{ }^{\circ} \mathrm{C}\right.$; left; vertically shifted in steps of $1.5 \times 10^{3} \mathrm{~cm}^{3} \mathrm{~g}^{-1}$ ) of CMK- 5 carbon before and after oxidative treatment with persulfate solution up to $10 \mathrm{~h}$. 
isotherm) and the pore emptying takes place at $c a . p / p_{0}=0.76$ (desorption isotherm). These values gradually shift towards lower relative pressures for the samples that underwent oxidative treatment for increasing duration. The material treated for $10 \mathrm{~h}$ shows pore filling and emptying at $c a . p / p_{0}=0.80$ and $p / p_{0}=0.66$, respectively. These data confirm that the pore surface polarity indeed depends on the oxidative treatment. Creating oxygen surface functions renders the pore walls more hydrophilic. In summary, the characterization of the silica-free CMK-5 carbon materials shows that the polar surface functions created by oxidative treatment increase the surface hydrophilicity. However, these surface functions only exist at the walls of the intra-tubular pores. The inter-tubular pore walls are untreated and, thus, hydrophobic. This is an example of a material with dual functionality; the mesoporous carbon materials combine hydrophobic pores with pores that are rather hydrophilic.

The effect of surface functionalization on the hydrophilicity must be expected to be more pronounced in the CMK-5@SBA15 carbon/silica composite materials, i.e. before removal of the silica, because at this stage the inter-tubular pores are still blocked and, therefore, do not contribute to the overall hydrophobicity. Fig. 4 (left) shows the respective $\mathrm{N}_{2}$ physisorption isotherms of all samples; the pore size distribution plots are shown in Fig. 4 (right). All samples show IV(a)-type isotherms, similar to the silica-free samples (Fig. 2), but with significantly lower overall $\mathrm{N}_{2}$ uptake, corresponding to a lower pore volume (see Table 1). The pore size distribution peak is centered at $c a$. $3.5 \mathrm{~nm}$. This peak represents the intra-tubular pores; it was also present in Fig. 2b (silica-free CMK-5 carbon samples) where it was only weakly resolved (visible as a shoulder) from the peak corresponding to the inter-tubular pores. (A second, much weaker peak at a pore size of $c a .7 .3 \mathrm{~nm}$ is also observed in all samples in Fig. 4b; it is absent in the silica-free samples, Fig. 2b, and originates most likely from residual mesopores in the SBA15 silica matrix that are not filled with carbon.) Oxidative treatment leads to a decrease of the specific pore volume to $\mathrm{ca}$. $73 \%$ of its initial value (from $0.33 \mathrm{~cm}^{3} \mathrm{~g}^{-1}$ to $0.24 \mathrm{~cm}^{3} \mathrm{~g}^{-1}$ ); the
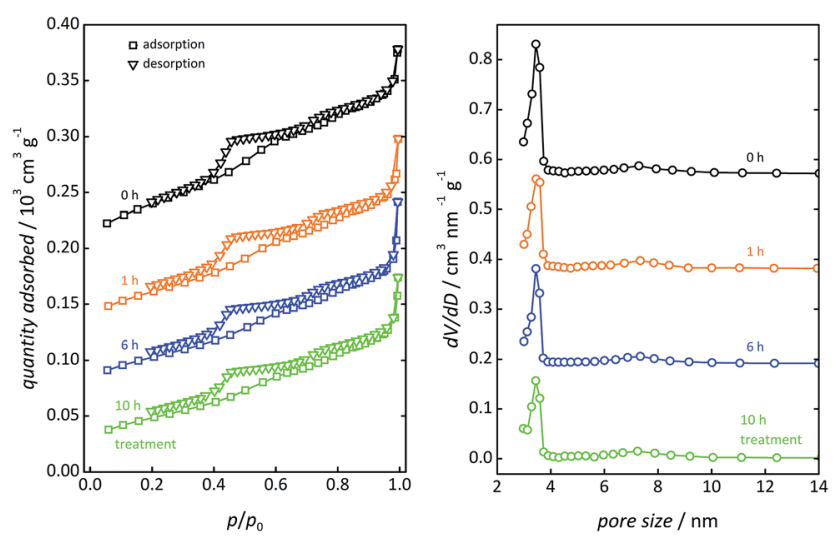

Fig. $4 \mathrm{~N}_{2}$ physisorption isotherms (left; vertically shifted in steps of $0.05 \times 10^{3} \mathrm{~cm}^{3} \mathrm{~g}^{-1}$ each) and $\mathrm{BJH}$ pore size distribution curves (right) of the CMK-5aSBA-15 carbon/silica composite materials before and after oxidative treatment with persulfate solution up to $10 \mathrm{~h}$.
BET surface area reduces to $c a$. $59 \%$ (from $273 \mathrm{~m}^{2} \mathrm{~g}^{-1}$ to 160 $\left.\mathrm{cm}^{2} \mathrm{~g}^{-1}\right)$. The average pore size, however, does not change significantly and the pore size distribution does not become any broader. These findings are in line with the respective data for the silica-free samples. The relative decrease in pore volume and BET surface area is more pronounced here because of the altogether lower initial values.

Fig. 5 shows the water sorption isotherms for the same samples as in Fig. 4; the isotherms are of type V, similar as for the respective silica-free samples. As in case of $\mathrm{N}_{2}$ sorption, the overall uptake of water is much lower than for the silica-free materials (Fig. 3), corresponding to a lower pore volume (see Table 1) owing to the absence of inter-tubular pores. As expected, the impact of oxidative treatment is much more visible in this series of samples than in case of the silica-free materials. For the pristine (i.e. non-treated) CMK-5@SBA-15 composite material the filling of the mesopores (adsorption isotherm) occurs in the region of $p / p_{0}=0.7-0.9$. During desorption two distinct steps are observed. The first step occurs at $c a . p / p_{0}=0.8-0.7$; it is present in the same pressure range for all samples after oxidative treatment and can be explained by the emptying of residual mesopores in the SBA-15 silica, not filled with carbon (as already observed by $\mathrm{N}_{2}$ sorption analysis). The second step in the desorption isotherm occurs at $c a . p / p_{0}=0.56-0.43$, corresponding to the emptying of the intra-tubular carbon pores. (This step was already present in the silica-free samples, but less obvious to the naked eye due to the larger scale in Fig. 3. This is shown in the ESI section in Fig. S2. $\dagger$ Interestingly, the step is weaker in the silica-free samples than in the silica-containing samples which cannot be explained in a straightforward way. Apparently, the emptying of the intra-tubular pores seems to be affected by the presence or absence of silica in the adjacent inter-tubular pores. This

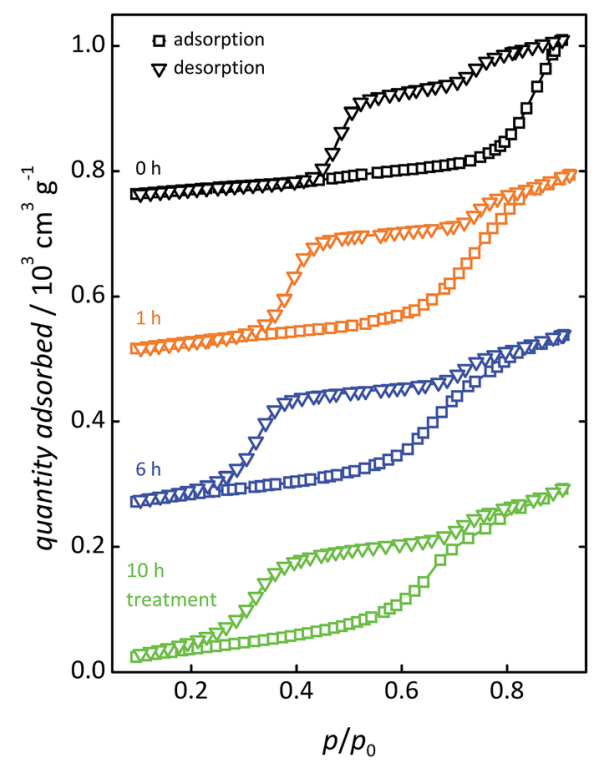

Fig. 5 Water vapor physisorption isotherms $\left(20^{\circ} \mathrm{C}\right.$; vertically shifted in steps of $0.25 \times 10^{3} \mathrm{~cm}^{3} \mathrm{~g}^{-1}$ each) of the CMK-5aSBA-15 carbon/silica composite materials before and after oxidative treatment with persulfate solution up to $10 \mathrm{~h}$. 
phenomenon is currently subject to more elaborate analysis in our lab.) This step is shifted significantly towards lower relative pressure upon oxidative treatment for increasing amounts of time; after treatment for 10 hours it occurs at $p / p_{0}=0.40-0.23$. These findings clearly show that the intra-tubular carbon surface is strongly affected by the oxidative treatment. The presence of oxygen surface functions (carbonyl and carboxyl groups) leads to strongly increased polarity; the carbon pore surface becomes more hydrophilic which explains why water is released from the intra-tubular pores during desorption at significantly lower relative pressure. ${ }^{4-50}$ The increase in hydrophilicity is supported by the fact that the quotient of the pore volume calculated from $\mathrm{N}_{2}$ physisorption divided by the value from $\mathrm{H}_{2} \mathrm{O}$ sorption increases with increasing oxidative treatment (see ESI section, Table $\mathrm{S} 1 \dagger$ ). This is attributed to the fact that water has a lower density in confined hydrophobic space compared to a hydrophilic surrounding. ${ }^{47,51}$

\section{Experimental}

\section{Synthesis of SBA-15 silica}

SBA-15 silica was prepared by a modified literature procedure. $^{7}$ A P-123 stock solution was prepared by mixing Pluronic P-123 block co-polymer (167.0 g, Sigma-Aldrich) in water (833.0 g) and stirring overnight. The P-123 stock solution (96.0 g) was diluted with water $(385 \mathrm{~mL})$, acidified with hydrochloric acid $(48 \mathrm{~mL}$, Stockmeier, $37 \%$ ), and stirred at $35{ }^{\circ} \mathrm{C}$ for one hour. Tetraethyl orthosilicate $(37.0 \mathrm{~mL}, \mathrm{ABCR}, 99 \%)$ was added and the solution was stirred for $24 \mathrm{~h}$ at $35{ }^{\circ} \mathrm{C}$. The resulting sol was transferred into a glass-lined autoclave and hydrothermally treated at $140{ }^{\circ} \mathrm{C}$ for $24 \mathrm{~h}$. After cooling down to room temperature the product was filtered off, washed with deionized water and dried at $120^{\circ} \mathrm{C}$ overnight. Finally the powder was calcined in a tube furnace for $6 \mathrm{~h}$ with a heating ramp of $2.5{ }^{\circ} \mathrm{C} \min ^{-1}$ at $550{ }^{\circ} \mathrm{C}$ under flowing air.

\section{Synthesis of CMK-5@SBA-15 composite}

A composite material of CMK-5 carbon within the pores of SBA-15 (CMK-5@SBA-15) was prepared by a modified literature procedure. $^{52}$ Oxalic acid dihydrate $(180 \mathrm{mg}$, ABCR, 98\%) was dissolved in furfuryl alcohol $(20.0 \mathrm{~mL}, \mathrm{ABCR}, 98 \%)$. This solution was mixed with SBA-15 silica to fill the silica pores with a theoretical loading of $130 \%$ of the pore volume (incipient wetness) by intensive grinding and subsequent thermal treatment at $60{ }^{\circ} \mathrm{C}$ for $24 \mathrm{~h}$ and $90{ }^{\circ} \mathrm{C}$ for $48 \mathrm{~h}$ in a drying cabinet. Carbonization was completed in a tube furnace at a temperature up to $850{ }^{\circ} \mathrm{C}$ (heating to $150{ }^{\circ} \mathrm{C}$ at $2{ }^{\circ} \mathrm{C} \mathrm{min}{ }^{-1}$ and maintaining at $150{ }^{\circ} \mathrm{C}$ for $3 \mathrm{~h}$, further heating to $300{ }^{\circ} \mathrm{C}$ at $1{ }^{\circ} \mathrm{C} \mathrm{min}{ }^{-1}$ and to $850{ }^{\circ} \mathrm{C}$ at $5{ }^{\circ} \mathrm{C} \min ^{-1}$ and maintaining at $850{ }^{\circ} \mathrm{C}$ for $4 \mathrm{~h}$ ) under vacuum.

\section{Oxidative treatment of the intra-tubular pores of CMK-5}

$2.30 \mathrm{~g}$ of the CMK-5@SBA-15 composite material was dispersed in $63 \mathrm{~mL}$ of an acidic solution of ammonium persulfate, $\left(\mathrm{NH}_{4}\right)_{2} \mathrm{~S}_{2} \mathrm{O}_{8}$, and stirred for $1 \mathrm{~h}, 6 \mathrm{~h}$ or $10 \mathrm{~h}$, respectively. The persulfate solution was prepared by mixing $33.5 \mathrm{~mL}$ concentrated sulfuric acid (96\%, Stockmeier) with $300 \mathrm{~mL}$ water, followed by addition of $\left(\mathrm{NH}_{4}\right)_{2} \mathrm{~S}_{2} \mathrm{O}_{8}(119.8 \mathrm{~g}$, ABCR, 98\%) and further stirring for 10 minutes. The silica/carbon composite samples were denoted as CMK-5@SBA-15(X), where $X$ represents the duration of previous persulfate treatment (in hours).

\section{Removal of the silica matrix}

The CMK-5@SBA-15 $(X)$ composite materials were treated in aqueous hydrofluoric acid $(12 \%, 100 \mathrm{~mL}$, Sigma-Aldrich) for at least $16 \mathrm{~h}$, and washed with deionized water. This procedure was repeated eight times to minimize the residual amount of silica. The silica-free carbon samples were denoted as CMK-5(X), where $X$ represents the duration of previous persulfate treatment (in hours).

\section{Characterization}

$\mathrm{N}_{2}$ physisorption analysis was conducted with a Quantachrome Autosorb 6 apparatus. Samples were degassed at $120^{\circ} \mathrm{C}$ for $12 \mathrm{~h}$. Pore size distributions were calculated by $\mathrm{BJH}$ model ${ }^{53}$ from the desorption branch of the isotherms. The BET surface areas $^{54}$ were calculated in a pressure range of $0.1 \leq p / p_{0} \leq 0.3$. Microporosity was estimated by the $t$-plot method. Total pore volumes were derived from the second-to-last data point in the adsorption isotherm $\left(c a . p / p_{0}=0.99\right)$. Water vapor sorption was performed on a Micromeritics 3Flex apparatus at $20{ }^{\circ} \mathrm{C}$. Samples were degassed at $120{ }^{\circ} \mathrm{C}$ for $12 \mathrm{~h}$. X-ray diffraction was carried out on a Bruker AXS D8 Advance diffractometer with $\mathrm{Cu} \mathrm{K}_{\alpha}$ radiation $(40 \mathrm{kV}, 40 \mathrm{~mA})$ with a step size of $2 \theta=0.0075^{\circ}$ and a counting time of $3 \mathrm{~s}$ per step. FT-IR spectra were measured on a Bruker Vertex 70 in transmission mode ( $\mathrm{KBr}$ matrix). Elemental analysis was carried out with an Elementar vario MicroCube.

\section{Conclusions}

Bimodal mesoporous CMK-5 carbon was modified by oxidative treatment selectively by temporary blocking of one of the two pore modes. Our data demonstrate that water vapor physisorption analysis provides a versatile method of distinguishing the two distinct modes of mesopores in CMK-5 carbon after selective modification of pore mode. The pore surface polarity serves as a probe for the increasing quantity of oxygen-containing functionalities formed by oxidative treatment for increased duration. After 10 hours of treatment the carbon contains $13.0 \mathrm{wt} \%$ oxygen while untreated CMK-5 carbon contains only $3.8 \mathrm{wt} \%$ oxygen.

\section{Acknowledgements}

C. W. thanks the Germany Fonds der Chemischen Industrie for a PhD Fellowship.

\section{Notes and references}

1 F. Schüth, Angew. Chem., Int. Ed., 2003, 42, 3604.

2 A.-H. Lu and F. Schüth, Adv. Mater., 2006, 18, 1793. 
3 H. Yang and D. Zhao, J. Mater. Chem., 2005, 15, 1217.

4 M. Tiemann, Chem. Mater., 2007, 20, 961.

5 J. Lee, J. Kim and T. Hyeon, Adv. Mater., 2006, 18, 2073.

6 C. Liang, Z. Li and S. Dai, Angew. Chem., Int. Ed., 2008, 47, 3696.

7 D. Zhao, Q. Huo, J. Feng, G. F. Chmelka and G. D. Stucky, J. Am. Chem. Soc., 1998, 120, 6024.

8 S. Jun, S. H. Joo, R. Ryoo, M. Kruk, M. Jaroniec, Z. Liu, T. Ohsuna and O. Terasaki, J. Am. Chem. Soc., 2000, 122, 10712.

9 S. H. Joo, S. J. Choi, I. Oh, J. Kwak, Z. Liu, O. Terasaki and R. Ryoo, Nature, 2001, 412, 169.

10 S. Che, K. Lund, T. Tatsumi, S. Iijima, S. H. Joo, R. Ryoo and O. Terasaki, Angew. Chem., Int. Ed., 2003, 42, 2182.

11 H. Zhou, S. Zhu, M. Hibino, I. Honma and M. Ichihara, Adv. Mater., 2003, 15, 2107.

12 X. Ji, K. T. Lee and L. F. Nazar, Nat. Mater., 2009, 8, 500.

13 J. Fan, T. Wang, C. Yu, B. Tu, Z. Jiang and D. Zhao, Adv. Mater., 2004, 16, 1432.

14 J. Lee, S. Yoon, T. Hyeon, S. M. Oh and K. B. Kim, Chem. Commun., 1999, 2177.

15 H. Zhou, S. Zhu, M. Hibino and I. Honma, J. Power Sources, 2003, 122, 219.

16 A. Vinu, C. Streb, V. Murugensan and M. Hartmann, J. Phys. Chem. B, 2003, 107, 8297.

17 M. Hartmann, A. Vinu and G. Chandrasekar, Chem. Mater., 2005, 17, 829.

18 J. H. Knox, B. Kaur and G. R. Millward, J. Chromatogr., 1986, $352,3$.

19 Z. Li and M. Jaroniec, Anal. Chem., 2004, 76, 5479.

20 C. Weinberger, S. Haffer, T. Wagner and M. Tiemann, Eur. J. Inorg. Chem., 2014, 2787.

21 W. Shen and W. Fan, J. Mater. Chem. A, 2013, 1, 999.

22 L. Liu, Y.-P. Zhu, M. Su and Z.-Y. Yuan, ChemCatChem, 2015, 7, 2765.

23 W. S. Ahn, K. I. Min, Y. M. Chung, H.-K. Rhee, S. H. Joo and R. Ryoo, Stud. Surf. Sci. Catal., 2002, 313, 2418.

24 A.-H. Lu, W. Schmidt, N. Matoussevitch, H. Bönnemann, B. Spliethoff, B. Tesche, E. Bill, W. Kiefer and F. Schüth, Angew. Chem., Int. Ed., 2004, 43, 4303.

25 A.-H. Lu, J.-J. Nitz, M. Comotti, C. Weidenthaler, K. Schlichte, C. W. Lehmann, O. Terasaki and F. Schüth, J. Am. Chem. Soc., 2010, 132, 14152.

26 D. Gu, W. Li, F. Wang, H. Bongard, B. Spliethoff, W. Schmidt, C. Weidenthaler, Y. Xia, D. Zhao and F. Schüth, Angew. Chem., Int. Ed., 2015, 54, 7060.

27 F. de Clippel, M. Dusselier, R. Van Rompaey, P. Vanelderen, J. Dijkmans, E. Makshina, L. Giebeler, S. Oswald, G. V. Baron, J. F. M. Denayer, P. P. Pescarmona, P. A. Jacobs and B. F. Sels, J. Am. Chem. Soc., 2012, 134, 10089.

28 B. Chang, J. Fu, Y. Tian and X. Dong, J. Phys. Chem. C, 2013, $117,6252$.
29 T. Pinto, P. Arquilliere, G. P. Niccolai, F. Lefebvre and V. Dufaud, New J. Chem., 2015, 39, 5300.

30 M. Liu, B. Liu, L. Liang, F. Wang, L. Shi and J. Sun, J. Mol. Catal. A: Chem., 2016, 418-419, 78.

31 X. Wang and R. Rinaldi, Catal. Today, 2016, 269, 48.

32 P. T. Huyen, M. Krivec, M. Kočevar, I. C. Bucur, C. Rizescu and V. I. Parvulescu, ChemCatChem, 2016, 8, 1146.

33 F. Wang, J. Xu, X. Shao, X. Su and Y. Huang, ChemSusChem, 2016, 9, 246.

34 W. Yin, Z. Tang, R. H. Venderbosch, Z. Zhang, C. Cannilla, G. Bonura, F. Frusteri and H. J. Heeres, ACS Catal., 2016, 6, 4411.

35 H. Nur, S. Ikeda and B. Ohtani, Chem. Commun., 2000, 2235. 36 H. Nur, S. Ikeda and B. Ohtani, J. Braz. Chem. Soc., 2004, 15, 719.

37 B. Karimi, H. M. Mirzaei, H. Behzadnia and H. Vali, ACS Appl. Mater. Interfaces, 2015, 7, 19050.

38 D. H. Paull, C. J. Abraham, M. T. Scerba, E. Alden-Danforth and T. Lectka, Acc. Chem. Res., 2008, 41, 655-663.

39 F. D. Kong, J. Liu, A. X. Ling, Z. Q. Xu, H. Y. Wang and Q. S. Kong, J. Power Sources, 2015, 299, 170.

40 A.-H. Lu, W. C. Li, N. Muratova, B. Spliethoff and F. Schüth, Chem. Commun., 2005, 5184.

41 P. A. Bazula, A.-H. Lu, J. J. Nitz and F. Schüth, Microporous Mesoporous Mater., 2008, 108, 266.

42 J. Roggenbuck, T. Waitz and M. Tiemann, Microporous Mesoporous Mater., 2008, 113, 575.

43 T. G. Ros, A. J. van Dillen, J. W. Geus and D. C. Koningsberger, Chem.-Eur. J., 2002, 8, 1151.

44 S. Biniak, G. Szymanski, J. Siedlewski and A. Swiatkowski, Carbon, 1997, 35, 1799.

45 H. P. Boehm, Carbon, 2002, 40, 145.

46 M. Thommes, K. Kaneko, A. V. Neimark, J. P. Olivier, F. Rodrigued-Reinoso, J. Roquerol and K. S. W. Sing, Pure Appl. Chem., 2015, 87, 1051.

47 M. Thommes, J. Morell, K. A. Cychosz and M. Fröba, Langmuir, 2013, 29, 14893.

48 T. Ohba, H. Kanoh and K. Kaneko, J. Phys. Chem. C, 2004, 108, 14964.

49 T. Horikawa, T. Sekida, J. Hayashi, M. Katoh and D. D. Do, Carbon, 2011, 49, 416.

50 T. Kimura, H. Kanoh, T. Kanda, T. Ohkubo, Y. Hattori, Y. Higaonna, R. Denoyel and K. Kaneko, J. Phys. Chem. B, 2004, 108, 14043.

51 L.-B. Sun, Y.-H. Sun, X.-D. Liu, L. Zhu and X.-Q. Liu, Curr. Org. Chem., 2014, 18, 1296.

52 A.-H. Lu, W. C. Li, W. Schmidt, W. Kiefer and F. Schüth, Carbon, 2004, 42, 2939.

53 E. P. Barrett, L. G. Joyner and P. P. Halenda, J. Am. Chem. Soc., 1951, 73, 373.

54 S. Brunauer, P. H. Emmett and E. Teller, J. Am. Chem. Soc., 1938, 60, 309. 